\title{
Siglenverzeichnis der zitierten Goethe-Ausgaben
}

FA Goethe, Johann Wolfgang. Sämtliche Werke. Briefe, Tagebücher und Gespräche. 40 Bde. Hg. Hendrik Birus et al. Frankfurt a. M.: Deutscher Klassiker Verlag, 1987-2013 (Frankfurter Ausgabe).

GB Goethe, Johann Wolfgang. Briefe. Historisch-kritische Ausgabe. Hg. im Auftrag der Klassik Stiftung Weimar, Goethe- und Schiller-Archiv, von Georg Kurscheidt, Norbert Oellers und Elke Richter. Berlin: Akademie Verlag / De Gruyter: Berlin und Boston, 2008-.

GT Goethe, Johann Wolfgang. Tagebücher. Historisch-kritische Ausgabe. Hg. Jochen Golz, unter Mitarbeit von Wolfgang Albrecht et al. Stuttgart und Weimar: Metzler, 1998-.

HA Goethe, Johann Wolfgang. Werke. 14 Bde. Hg. Erich Trunz. Hamburg und München: Wegner, 1948-1964 (Hamburger Ausgabe).

LA Goethe. Die Schriften zur Naturwissenschaft. Vollständige mit Erläuterungen versehene Ausgabe im Auftrage der Deutschen Akademie der Naturforscher Leopoldina. Hg. Dorothea Kuhn und Wolf von Engelhardt, Weimar: Hermann Böhlaus Nachfolger, 19472019 (Leopoldina-Ausgabe).

MA Goethe, Johann Wolfgang. Sämtliche Werke nach Epochen seines Schaffens. 21 Bde. (in 33). Hg. Karl Richter in Zusammenarbeit mit Herbert G. Göpfert, Norbert Miller, Gerhard Sauder und Edith Zehm. München: Hanser, 1985-1998 (Münchner Ausgabe).

WA Goethe. Werke. Hg. im Auftrag der Großherzogin Sophie von Sachsen. 143 Bde, Weimar: Hermann Böhlaus Nachfolger, 1887-1913. Nachdruck München: DTV, 1987 (Weimarer Ausgabe). 
